\title{
Multiple injections of human umbilical cord-derived mesenchymal stromal cells through the tail vein improve microcirculation and the microenvironment in a rat model of radiation myelopathy
}

Li Wei ${ }^{1,2^{*}+}$, Jing Zhang ${ }^{1,6+}$, Xiu-Bin Xiao ${ }^{1+}$, Hai-Xing Mai ${ }^{1}$, Ke Zheng $^{3}$, Wan-Liang Sun ${ }^{1,7}$, Lei Wang ${ }^{4}$, Feng Liang ${ }^{1}$, Zai-Liang Yang ${ }^{5}$, Yuan Liu ${ }^{5}$, Yan-Qing Wang ${ }^{1}$, Zhi-Fang Li ${ }^{1}$, Jia-Ning Wang ${ }^{4}$, Wei-Jing Zhang ${ }^{1 *}$ and Hua You ${ }^{1 *}$

\begin{abstract}
Background: At present, no effective clinical treatment is available for the late effects of radiation myelopathy. The aim of the present study was to assess the therapeutic effects of human umbilical cord-derived mesenchymal stromal cells (UC-MSCs) in a rat model of radiation myelopathy.

Methods: An irradiated cervical spinal cord rat model was generated. UC-MSCs were injected through the tail vein at 90, 97, 104 and 111 days post-irradiation. Behavioral tests were performed using the forelimb paralysis scoring system, and histological damage was examined using Nissl staining. The microcirculation in the spinal cord was assessed using von Willebrand factor (VWF) immunohistochemical analysis and laser-Doppler flowmetry. The microenvironment in the spinal cord was determined by measuring the pro-inflammatory cytokines interleukin-1 $\beta$ (IL-1 $\beta)$ and tumor necrosis factor- $\alpha(T N F-a)$ in the serum and the anti-inflammatory cytokines brain-derived neurotrophic factor (BDNF) and glial cell-derived neurotrophic factor (GDNF) in the spinal cord.

Results: Multiple injections of UC-MSCs through the tail veil decreased the forelimb paralysis, decreased spinal cord histological damage, increased the number of neurons in the anterior horn of the spinal cord, increased the endothelial cell density and the microvessel density in the white matter and gray matter of the spinal cord, increased the relative magnitude of spinal cord blood flow, down-regulated pro-inflammatory cytokine expression in the serum, and increased anti-inflammatory cytokine expression in the spinal cord.
\end{abstract}

Conclusion: Multiple injections of UC-MSCs via the tail vein in a rat model of radiation myelopathy significantly improved the microcirculation and microenvironment through therapeutic paracrine effects.

Keywords: Radiation myelopathy, Endothelial cell, Spinal cord blood flow, Paracrine system, Human umbilical cord-derived mesenchymal stromal cells

\footnotetext{
*Correspondence: weilikw@163.com; zhangwj3072@163.com;

youhua307@163.com

${ }^{\dagger}$ Equal contributors

${ }^{1}$ Affiliated Hospital of the Academy of Military Medical Sciences, No.8 East

Main Street, Fengtai District, Beijing 100071, China

${ }^{2}$ Key Laboratory of Birth Defects and Reproductive Health of the National

Health and Family Planning Commission, Chongqing Population and the

Family Planning Science and Technology Research Institute, Chongqing

400020, China

Full list of author information is available at the end of the article
}

( Biomed Central
(C) 2014 Wei et al.; licensee BioMed Central Ltd. This is an Open Access article distributed under the terms of the Creative Commons Attribution License (http://creativecommons.org/licenses/by/4.0), which permits unrestricted use, distribution, and reproduction in any medium, provided the original work is properly credited. The Creative Commons Public Domain Dedication waiver (http://creativecommons.org/publicdomain/zero/1.0/) applies to the data made available in this article, unless otherwise stated. 


\section{Introduction}

The spinal cord is a well-known example of "late-reacting" tissue in response to irradiation [1]. Exposure of the spinal cord to radiation can result in radiation myelopathy, which is a rare but serious complication of radiotherapy for cancer [2]. The late effects of radiation myelopathy (i.e., those occurring 6 months to several years after treatment) can be extremely severe and may seriously decrease the patient's quality of life. These effects are particularly dangerous because they are generally irreversible $[3,4]$. Currently, there is no effective clinical treatment for the late effects of radiation myelopathy.

Two distinct hypotheses-the glial hypothesis (demyelination) and the vascular hypothesis-have been developed to explain the underlying mechanisms leading to the development of selective white matter necrosis and, in particular, to identify the primary "target cell population" within the CNS [4]. Vascular damage is considered the key step in the development of radiation myelopathy [1,2,4-8]. Hence, the vascular endothelial cell population has been proposed as one of the most critical targets of the late effects of radiation myelopathy [9].

Previous studies have examined early endothelial cell apoptosis in the spinal cord after irradiation [10,11]. Radiation-induced apoptosis of endothelial cells has been shown to depend on the ASMase pathway rather than the p53 pathway [10]. Because we have clearly determined the time course of the endothelial cell response within 180 days post-irradiation and demonstrated its relationship with spinal cord blood flow [12], it seems possible that targeting endothelial cell death may be a neuroprotective strategy that can limit the late effects of spinal cord irradiation [12,13].

Recently, multipotent mesenchymal stromal cell (MSC) treatment has attracted special attention as a new alternative strategy for stimulating regeneration. MSCs are primitive cells originating from the mesodermal germ layer and are classically described as giving rise to connective tissues, skeletal muscle cells, and cells of the vascular system [14]. MSCs may exhibit immunosuppressive properties and have been suggested to be "immuneprivileged". They are thus protected from rejection, potentially permitting their use in allo-transplantation. It appears that MSCs have the capacity to localize to injured tissue and differentiate into specific cell types [15]. Some reports suggest that the therapeutic effects of MSCs in injured tissue are mainly mediated by paracrine activity, including the stimulation of endogenous repair, angiogenesis and arteriogenesis; attenuation of remodeling; and reduction of apoptosis [16]. Interestingly, injected murine MSCs, but not human MSCs, differentiated into osteosarcomas in injured lungs. Thus, human MSCs appear to be more feasible and safer for use than murine MSCs [17].
The aim of the present study was to assess the therapeutic effects of human umbilical cord-derived mesenchymal stromal cells (UC-MSCs) in a rat model of radiation myelopathy. To the best of our knowledge, this is the first clinically based translational study to assess and highlight human MSC therapy for the treatment of radiation myelopathy.

\section{Materials and methods}

\section{Animals}

Adult female Sprague-Dawley (SD) rats (160-200 g, Laboratory Animal Center, Academy of Military Medical Sciences, Beijing, China) were housed and cared for according to the guidelines for the care and use of laboratory animals of the NIH and Academy of Military Medical Sciences (Beijing, China). All experimental procedures were approved by the Committee for Animal Use at the Academy of Military Medical Sciences. Every effort was made to minimize the number of animals used as well as their suffering. Water and food were available ad libitum in the cages.

\section{Isolation and differentiation of human UC-MSCs}

UC-MSCs were isolated from Wharton's jelly (WJ) of umbilical cords according to previously described methods with some modification [18]. Fresh umbilical cords were collected after obtaining consent from the mothers. The umbilical cords were rinsed in phosphatebuffered saline (PBS) until the cord blood was cleared, and the blood vessels were removed. The remaining WJ tissue was cut into $1-2 \mathrm{~mm}^{3}$ pieces and placed in sixwell plates in the presence of $0.1 \%$ collagenase type II (Sigma, USA) in PBS at $37^{\circ} \mathrm{C}$ for $1 \mathrm{~h}$. Ten percent fetal bovine serum (FBS, Invitrogen, USA) was then added to stop the digestion. The dissociated mesenchymal cells were dispersed in 10\% FBS-DMEM and further cultured until well-developed colonies of the fibroblast-like cells reached $80 \%$ confluence. Then, the cultures were trypsinized with $0.25 \%$ trypsin-EDTA (Invitrogen, USA) and passaged into new flasks for further expansion. The multipotent differentiation capacity of the UC-MSCs was confirmed by their differentiation into adipocytes, chondroblasts and osteoblasts using Oil Red O staining (adipocytes), alcian blue staining (chondroblasts), and alkaline phosphatase (osteoblasts), respectively. The surface markers of the UC-MSCs were also examined by flow cytometry.

\section{Irradiation and UC-MSC injection}

A total of $20 \mathrm{SD}$ rats were randomly divided into 4 groups ( $n=5$ each): normal control (control) group, untreated irradiation (irradiation) group, PBS treatment (PBS) group, and UC-MSC treatment (UC-MSC) group. Each rat in the irradiation, PBS and UC- MSC groups was anesthetized 
using 3\% sodium pentobarbiturate $(45 \mathrm{mg} / \mathrm{kg})$ delivered by intraperitoneal injection and subsequently irradiated using a ${ }^{60} \mathrm{Co}$ source. A ${ }^{60} \mathrm{Co}$ irradiator (Model GWXJ80, NPIC, Chengdu, China) was used to conduct gamma ray irradiation, and the rats were irradiated with 30 Gy at a dose rate of approximately $150 \mathrm{cGy} / \mathrm{min}$. The beam was strictly limited to a $2-\mathrm{cm}$ segment of the cervical spine field spanning $\mathrm{C} 2-\mathrm{T} 2$. According to previous reports $[2,10,11,19]$, forelimb paralysis associated with white matter necrosis occurs approximately 20 weeks following the delivery of single doses of 20 Gy or more to the cervical spinal cord in this radiation myelopathy model. Single doses of 19.5 and 22 Gy represent the ED50 and ED100, respectively, for forelimb paralysis within 180 days secondary to white matter necrosis in the rat spinal cord model. Rats in the PBS and UC-MSC groups were given PBS and UC-MSC $\left(1 \times 10^{6}\right.$ cells $)$ injections through the tail vein in a $200 \mu \mathrm{l}$ volume at 90, 97, 104 and 111 days post-irradiation. Rats in the irradiation group were not provided any treatment after irradiation. Rats in the control group were fed normally and not irradiated during the same period. The rats were observed daily for up to 180 days post-irradiation.

\section{Assessment of motor function}

At 180 days post-irradiation, the motor function of five rats was examined using a slightly modified version of the methodology described by Chiang [20]. A technical assistant who was blinded to the experimental conditions evaluated the rats' motor function. In the subjective scoring system that was used, 0 represented total paralysis of both forelimbs, 1 represented definite sub-total paralysis (one forelimb paralyzed or slight movement of both forelimbs; toes do not spread when the animal is held up), 2 represented definite abnormal movement (very unsteady movements, low stance, uncoordinated movements, hops), 3 represented questionably abnormal movement (slightly wobbly or uncoordinated movements), and 4 represented normal movement.

\section{Assessment of spinal cord blood flow (SCBF)}

SCBF was measured using a MoorLab laser-Doppler flowmeter (MoorLab Instruments, Devon, England) as described in our previous study [12]. This instrument recorded the relative change in blood flow over time. Briefly, the animals were mounted on a stereotactic instrument equipped with a vertebral fixation device to stabilize the vertebral column. Body temperature was maintained at $37.0 \pm 0.5^{\circ} \mathrm{C}$ using a heating blanket. The laser-Doppler probe was affixed to a micromanipulator and placed perpendicular to the spinal cord, barely touching the dorsal surface of the dura mater. Laser-Doppler signal readings were recorded on a computer and were analyzed using Moorsoft for Windows (version 1.31).
SCBF was measured before irradiation $(0 \mathrm{~d})$ and $180 \mathrm{~d}$ after irradiation. The mean value before irradiation $(0 \mathrm{~h})$ was used as the basal value, and the final value for each rat at each later time point was expressed as a percentage of the basal value (100\%).

\section{Specimen processing and histology}

After the SCBF assessment at 180 days post-irradiation, blood samples were collected from the heart and allowed to clot for $2 \mathrm{~h}$ at room temperature. Serum was obtained after centrifugation at $2000 \mathrm{rpm}$ for $20 \mathrm{~min}$ at $4^{\circ} \mathrm{C}$ and stored at $-80^{\circ} \mathrm{C}$. The $\mathrm{C} 2-\mathrm{T} 2$ spinal cord segment was removed, and a 1-cm-long central section of this segment was cut and immersed in 4\% paraformaldehyde for histological and immunohistochemical analysis. The remaining portion of the spinal cord was frozen in dry ice power and stored at $-80^{\circ} \mathrm{C}$ prior to use. The previously mentioned 1-cm-long central section of the C2-T2 spinal cord segment was dehydrated in ethanol and embedded in paraffin. The sections (5 $\mu \mathrm{m}$ thickness) were deparaffinized, rehydrated, and stained using Nissl staining. For Nissl staining, the sections were incubated with $0.1 \%$ cresyl violet at $37^{\circ} \mathrm{C}$ for $10 \mathrm{~min}$ and subsequently dehydrated, mounted, and observed under a light microscope. Neurons with diameters $>20 \mu \mathrm{m}$ in both anterior horns of the spinal cord were counted.

\section{Immunohistochemical analysis}

The sections ( $5 \mu \mathrm{m}$ thickness) were subjected to microwave antigen retrieval using Tris-EDTA ( $\mathrm{pH} \mathrm{9,} 10 \mathrm{~min}$ ). Endogenous peroxidase was quenched using $3 \% \mathrm{H}_{2} \mathrm{O}_{2}$ for $10 \mathrm{~min}$. The sections were incubated with polyclonal rabbit anti-human von Willebrand factor (vWF, also called factor VIII-related antigen) antibody (1:100 dilution; DakoCytomation, Denmark) or anti-human nuclei antibody (MAB1281, 1:1000 dilution; Millipore) at $37^{\circ} \mathrm{C}$ for $3 \mathrm{~h}$. The primary antibody was omitted from the negative control. After washing with PBS, the sections were incubated with biotinylated monoclonal anti-rabbit IgG for $20 \mathrm{~min}$ at room temperature using a SABC Detection System (Boster Biological Technology, Wuhan, China) according to the manufacturer's protocol. The slides were developed with diaminobenzidine (DAB; DakoCytomation, Denmark), counterstained with Mayer's hematoxylin, dehydrated with increasing concentrations of alcohol, cleared in xylene, and mounted in neutral balsam (Sigma, USA).

\section{ELISA}

The levels of interleukin-1 $\beta$ (IL-1 $\beta$ ) and tumor necrosis factor- $\alpha$ (TNF- $\alpha)$ in the serum and the levels of brainderived neurotrophic factor (BDNF) and glial cellderived neurotrophic factor (GDNF) in the spinal cord tissues were determined using commercially available 
ELISA kits according to the manufacturer's protocols (IL-1 $\beta$ and TNF- $\alpha$, Invitrogen, Carlsbad, California, USA; BDNF, MyBioSource, SanDiego, California, USA; GDNF, Abnova, Taipei city, Taiwan). The OD value was determined by an ELISA reader at a wavelength of $450 \mathrm{~nm}$ and calculated in the linear part of the curve.

\section{Morphometric analysis}

For the Nissl staining, two sections per rat were selected in every sixth section, and a total of ten sections per time point were carefully examined at $100 \mathrm{X}$ magnification under an Olympus microscope [12]. According to the methodology described previously by $\mathrm{Li}$ et al. and our group [10-12], the endothelial cell density was defined as the total number of endothelial cells that contained a nucleus divided by the transverse cross-sectional area of the spinal cord, and the microvessel density was defined as the total number of microvessels that were transversely sectioned and contained either an endothelial cell nucleus or no nucleus divided by the transverse cross-sectional area of the spinal cord. One hotspot area in the gray matter and another hotspot area in the white matter were selected at 100X magnification. The densities of the endothelial cells and microvessels were then determined using two fixed fields $\left(0.768 \mathrm{~mm}^{2}\right)$ within each of these two areas at 400X magnification per section. Three sections per rat were selected in every sixth section, and a total of thirty fields per time point in the gray matter and thirty fields per time point in the white matter were used for the analysis. Image-ProPlus software was used for the analyses. For morphometric analysis, two blinded pathologists carefully and independently examined all selected sections. The number of neurons, the endothelial cell density and the microvessel density were determined in the individual analyses, and mean values were calculated. We used some data from our previous paper [12] as the same experimental conditions which were employed in the present work.

\section{Statistics}

All quantitative data are expressed as the mean value \pm SEM. Differences were evaluated using one-way ANOVA. Comparisons between values at two time points were performed using the least significant difference (LSD) procedure. A $P$ value $<0.05$ was considered statistically significant.

\section{Results}

\section{Characterization of UC-MSCs}

The UC-MSCs exhibited similar spindle- and fibroblastlike shapes (Figure 1A). The multipotent differentiation capacity of the UC-MSCs was confirmed by their differentiation into adipocytes, osteoblasts and chondroblasts, as shown by the staining of the in vitro differentiation cultures with Oil Red O (Figure 1B, adipocytes), alkaline phosphatase (Figure 1C, osteoblasts), and alcian blue (Figure 1D, chondroblasts). The surface markers of the UC-MSCs were examined by flow cytometry. Most of the cultured cells were positive for CD90, CD73 and CD105 expression but negative for CD45, CD34, CD14, CD19 and HLA-DR expression (Figure 1E-L).

\section{UC-MSC administration improved locomotion recovery}

To determine the potential benefit of the UC-MSC infusion on the spinal cord after irradiation, behavioral tests were performed using the forelimb paralysis scoring system. Although we did not observe total paralysis at 180 days post-irradiation in our radiation myelopathy rat model, in which demyelination did not occur, the irradiated rats still exhibited significant forelimb motor function impairment compared with the normal controls at 180 days post-irradiation [12]. In irradiated animals, UC-MSC treatment significantly decreased the forelimb paralysis, compared with PBS treatment and no treatment, indicating that irradiated animals treated with UC-MSCs exhibited improved motor function (indicated by a higher score on the test). There were no significant differences in the forelimb paralysis score between PBStreated irradiated animals and untreated irradiated animals (Figure 2).

\section{UC-MSC administration ameliorated irradiation-induced histological damage in the spinal cord}

Next, we evaluated whether UC-MSC injection could decrease histological damage to the spinal cord after irradiation. We previously determined that a single 30-Gy dose of irradiation primarily injured the neurons in the spinal cord. A markedly thickened cytoplasm, significant swelling and distension of the soma, and hazy Nissl bodies were observed in the neurons between 30 days and 90 days post-irradiation. After 90 days post-irradiation, this neuronal damage was reversed to some degree [12]. Histological examination of spinal cord sections using Nissl staining revealed neuronal damage similar to that observed in the untreated irradiated animals and in the PBS-treated animals at 180 days post-irradiation (Figure 3). Interestingly, the neurons of UC-MSC-treated irradiated animals were less damaged than those of untreated irradiated animals (Figure 3 ).

We also counted the number of neurons in the anterior horn of the spinal cord following irradiation using Nissl staining. The number of neurons in the UC-MSCtreated animals $(15.6 \pm 0.6)$ was significantly higher than that in the PBS-treated animals $(11.4 \pm 1.0)$ and that in the untreated irradiated animals $(11.5 \pm 1.8)$ at 180 days post-irradiation (Figure 4). The number of neurons in the PBS-treated animals was similar to that in the untreated irradiated animals (Figure 4). 


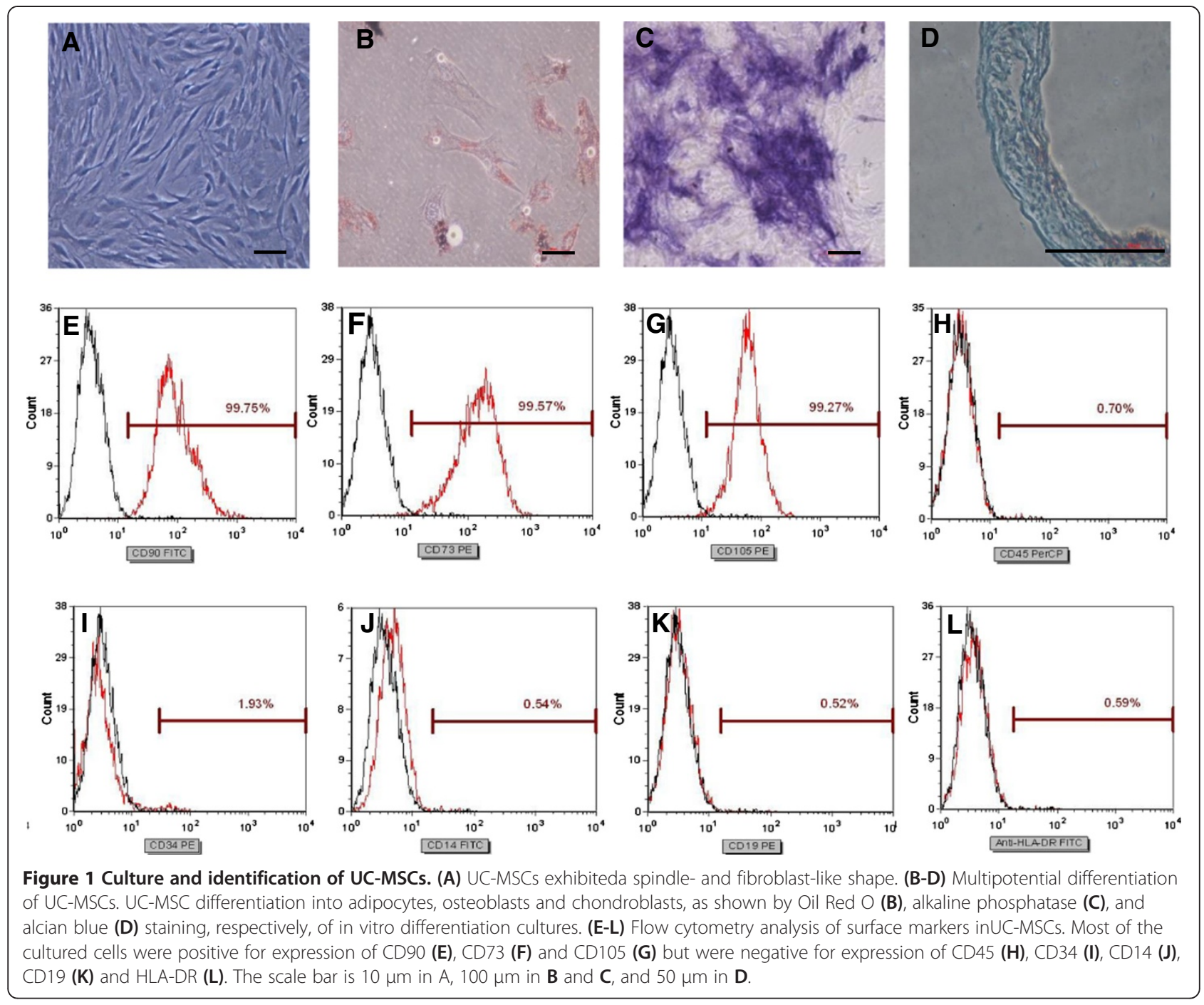

\section{UC-MSC administration improved irradiation-induced microcirculation dysfunction in the spinal cord}

To explore angiogenesis induced by UC-MSC administration, microvessels were identified with anti-factor VIII antibody, a specific marker for endothelial cells. The endothelial cell density and microvessel density were then quantified in the white matter and gray matter of the spinal cord (Figure 5). The endothelial cell and microvessel densities were significantly higher in the UC-MSCtreated animals [endothelial cell density: $(34.7 \pm 2.8) / \mathrm{mm}^{2}$ in the white matter, $(115.9 \pm 6.2) / \mathrm{mm}^{2}$ in the gray matter; microvessel density: $(69.9 \pm 4.6) / \mathrm{mm}^{2}$ in the white matter, $(255.2 \pm 7.2) / \mathrm{mm}^{2}$ in the gray matter] than those in the PBStreated animals [endothelial cell density: $(25.2 \pm 2.5) / \mathrm{mm}^{2}$ in the white matter, $(99.0 \pm 4.6) / \mathrm{mm}^{2}$ in the gray matter; microvessel density: $(56.0 \pm 3.5) / \mathrm{mm}^{2}$ in the white matter, $(237.4 \pm 9.1) / \mathrm{mm}^{2}$ in the gray matter] and those in the untreated irradiated animals [endothelial cell density $(24.7 \pm$ $3.0) / \mathrm{mm}^{2}$ in the white matter, $(99.0 \pm 5.3) / \mathrm{mm}^{2}$ in the gray matter; microvessel density $(56.9 \pm 4.5) / \mathrm{mm}^{2}$ in the white matter, $(229.6 \pm 9.6) / \mathrm{mm}^{2}$ in the gray matter] at 180 days post-irradiation (Figure 6). The endothelial cell and microvessel densities were similar in the PBS-treated animals and the untreated irradiated animals (Figure 6). We also used a marker of human nuclei, MAB1281, to identify surviving UC-MSCs in spinal cord sections by immunohistochemistry. However, UC-MSCs were not found in spinal cord sections of UC-MSC-treated animals (data not shown).

To address the functional effects of alterations in endothelialcell density and microvessel density, we applied laser-Doppler flowmetry to record the relative magnitude of the spinal cord blood flow [12]. The relative magnitude of the spinal cord blood flow in the UC-MSCtreated animals $(79.0 \pm 4.3)$ was significantly higher than that in the PBS-treated animals $(64.8 \pm 3.4)$ and untreated irradiated animals $(65.5 \pm 3.1) 180$ days post-irradiation (Figure 7). The relative magnitude of the spinal cord blood 


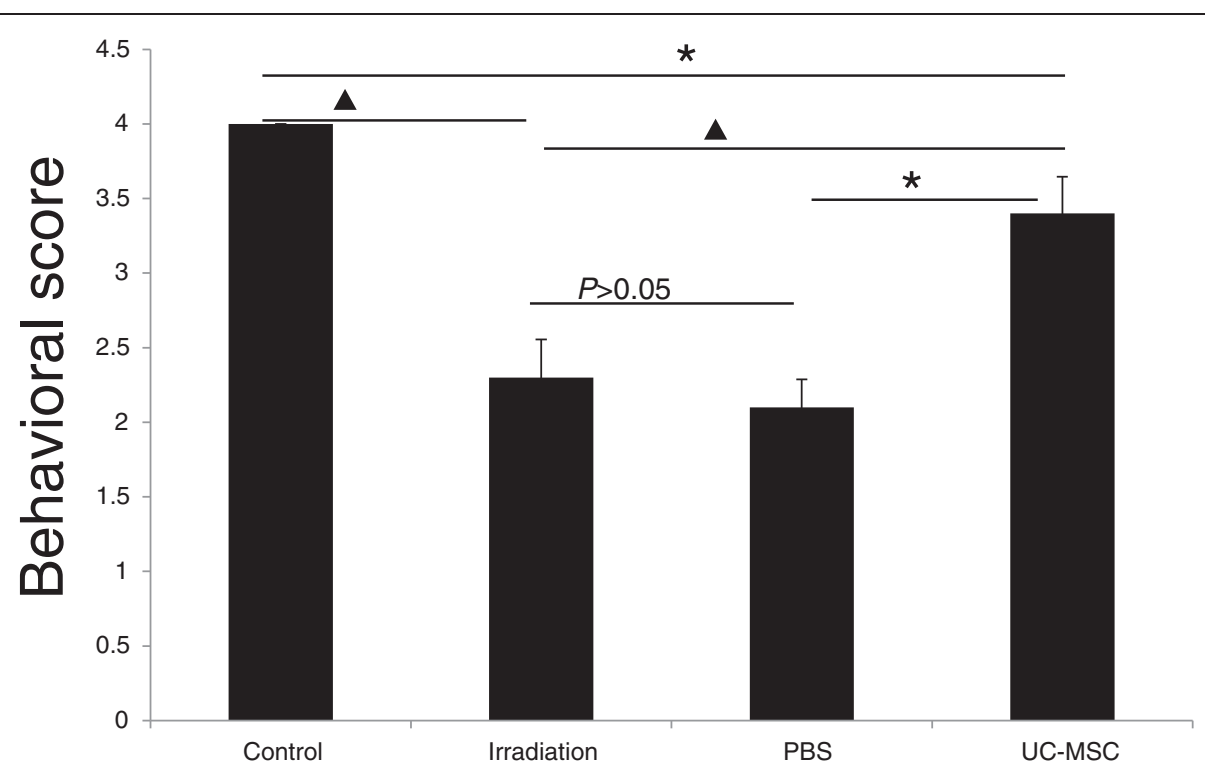

Figure 2 UC-MSC administration improved locomotion recovery at 180 days post-irradiation $\left(n=5,{ }^{*} P<0.05,{ }^{\Delta} P<0.01\right)$. Error bars represent the S.E.M.
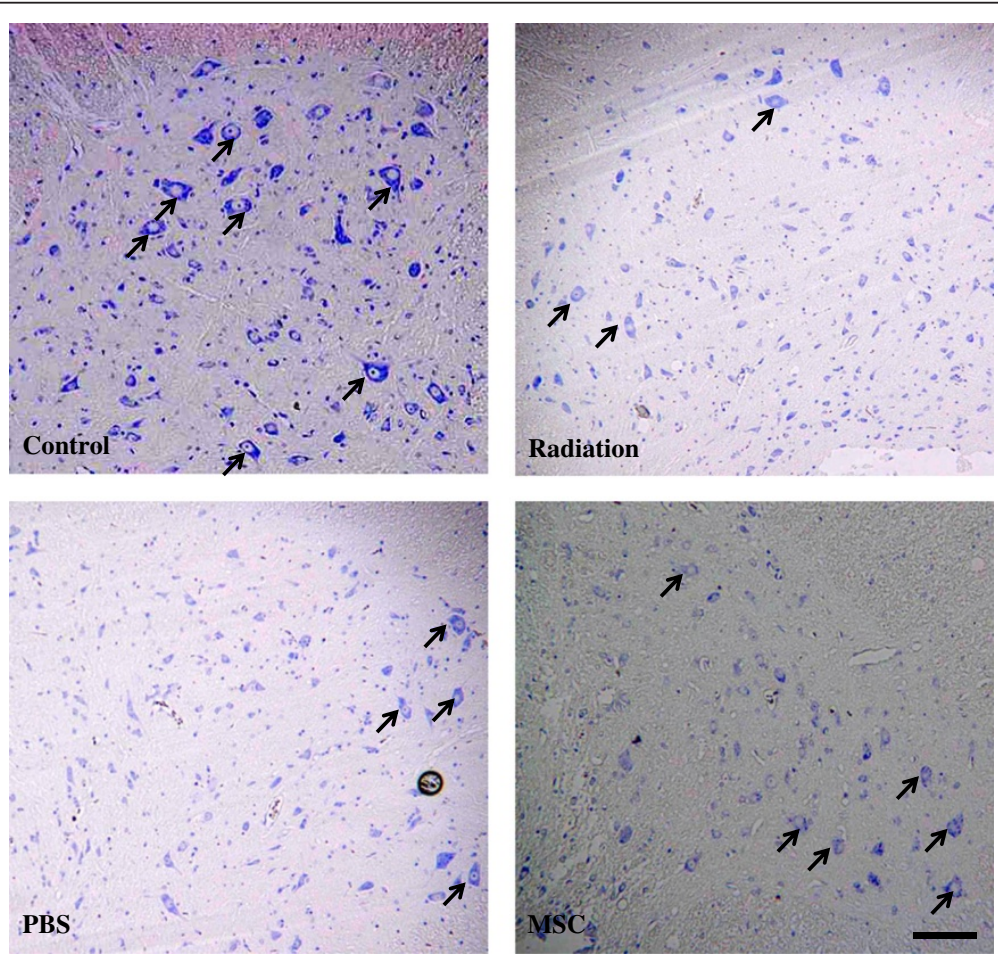

Figure 3 UC-MSC administration decreased irradiation-induced histological damage in the spinal cord. Representative micrographs of Nissl-stained rat spinal cords at 180 days post-irradiationfollowing systemic injection of UC-MSCs. Arrows indicate Nissl bodies. Scale bars $=100 \mu \mathrm{m}$. 


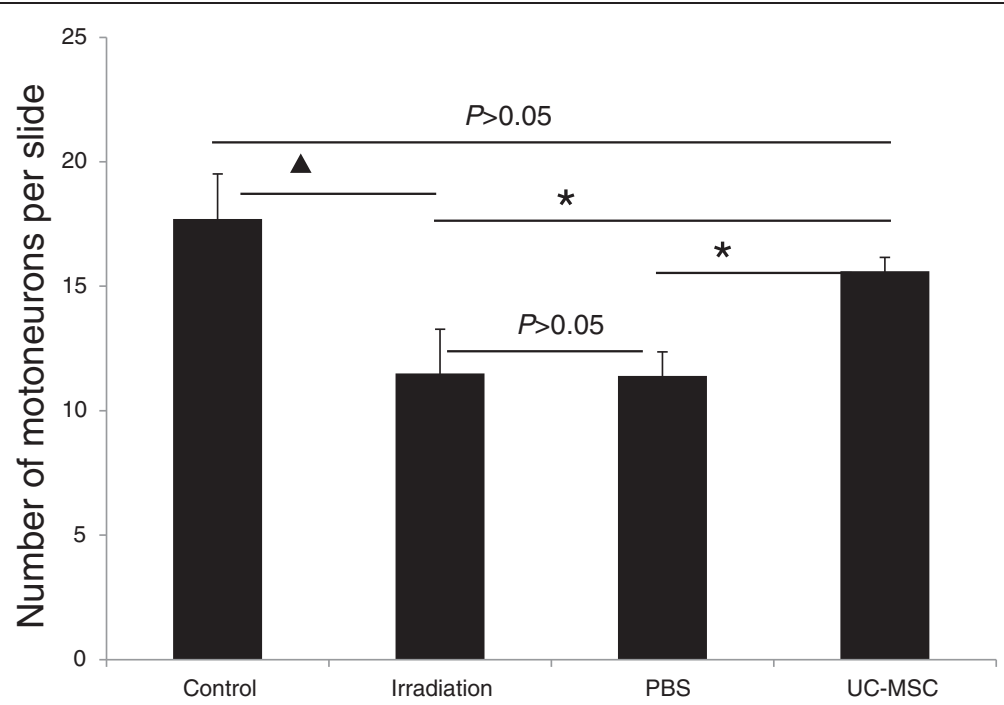

Figure 4 UC-MSC administration improved neuron survival in the anterior horn at 180 days post-irradiation $\left(\mathrm{n}=10,{ }^{*} P<0.05,{ }^{\Delta} P<0.01\right)$.

Error bars represent the S.E.M.

flow in the PBS-treated animals and the untreated irradiated animals was similar (Figure 7).

\section{UC-MSC administration reversed irradiation-induced inflammation}

We also determined whether the UC-MSC injection could reverse the spinal cord inflammation that arises after irradiation. We measured the levels of the proinflammatory cytokines IL-1 $\beta$ and TNF- $\alpha$ in the serum as well as the anti-inflammatory cytokines BDNF and
GDNF in the spinal cord tissues to assess the inflammatory status. The irradiated rats exhibited significantly higher levels of IL- $1 \beta(283.2 \pm 14.7 \mathrm{pg} / \mathrm{ml})$ and TNF- $\alpha$ $(118.2 \pm 5.4 \mathrm{pg} / \mathrm{ml})$ in the serum and lower levels of BDNF $(59.7 \pm 4.9 \mathrm{pg} / \mathrm{mg})$ and GDNF $(65.4 \pm 8.4 \mathrm{pg} / \mathrm{mg})$ in the spinal cord compared with the normal controls [IL-1 $\beta: 221.2 \pm 9.8 \mathrm{pg} / \mathrm{ml} ; \quad$ TNF- $\alpha: 92.8 \pm 5.0 \mathrm{pg} / \mathrm{ml}$; BDNF:106.4 $\pm 14.1 \mathrm{pg} / \mathrm{mg}$; GDNF:134.4 $\pm 13.6 \mathrm{pg} / \mathrm{mg}$ ] at 180 days post-irradiation (Figure 8). The levels of IL-1 $\beta$ $(235.4 \pm 5.2 \mathrm{pg} / \mathrm{ml})$ and TNF- $\alpha(100.5 \pm 4.4 \mathrm{pg} / \mathrm{ml})$ in the

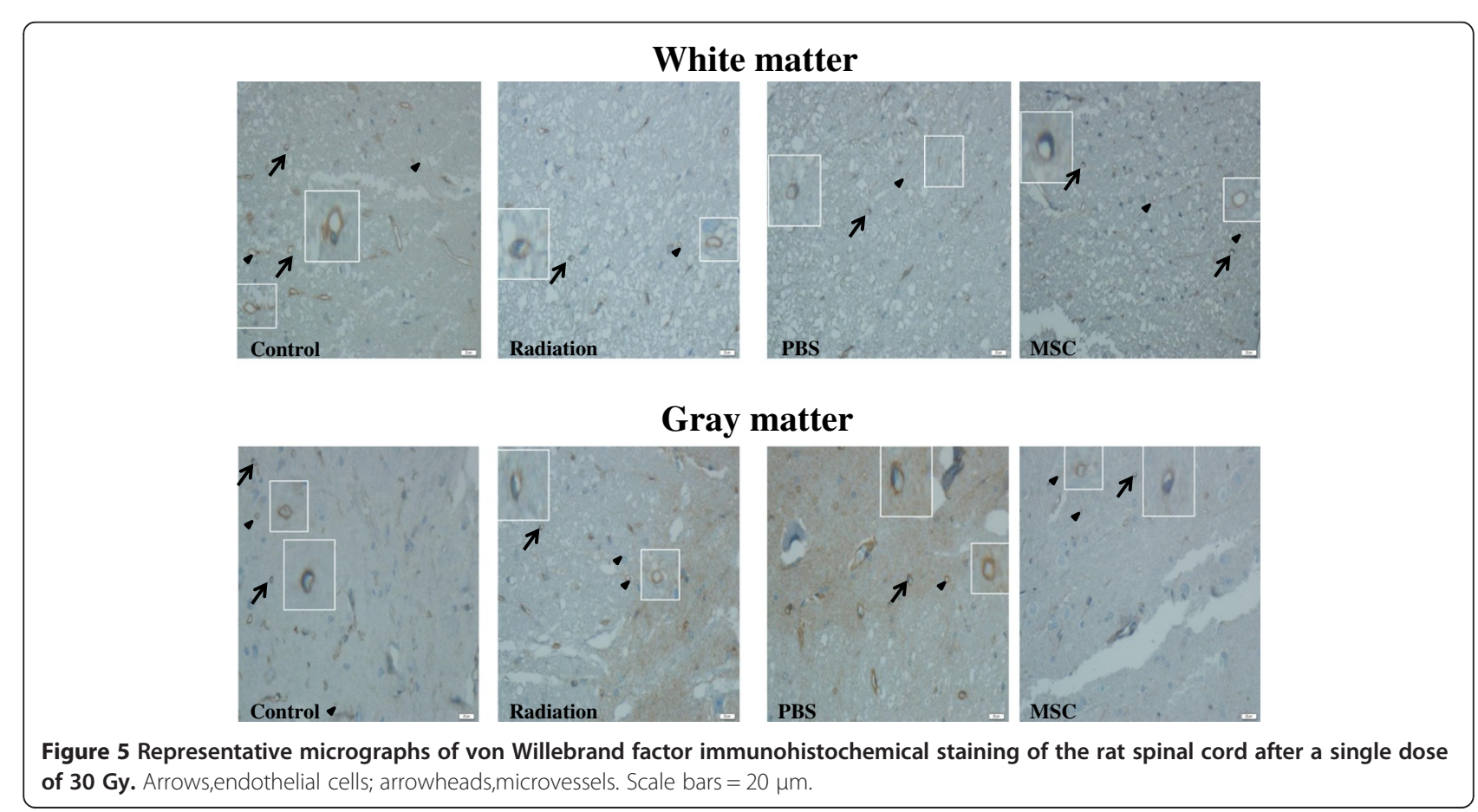




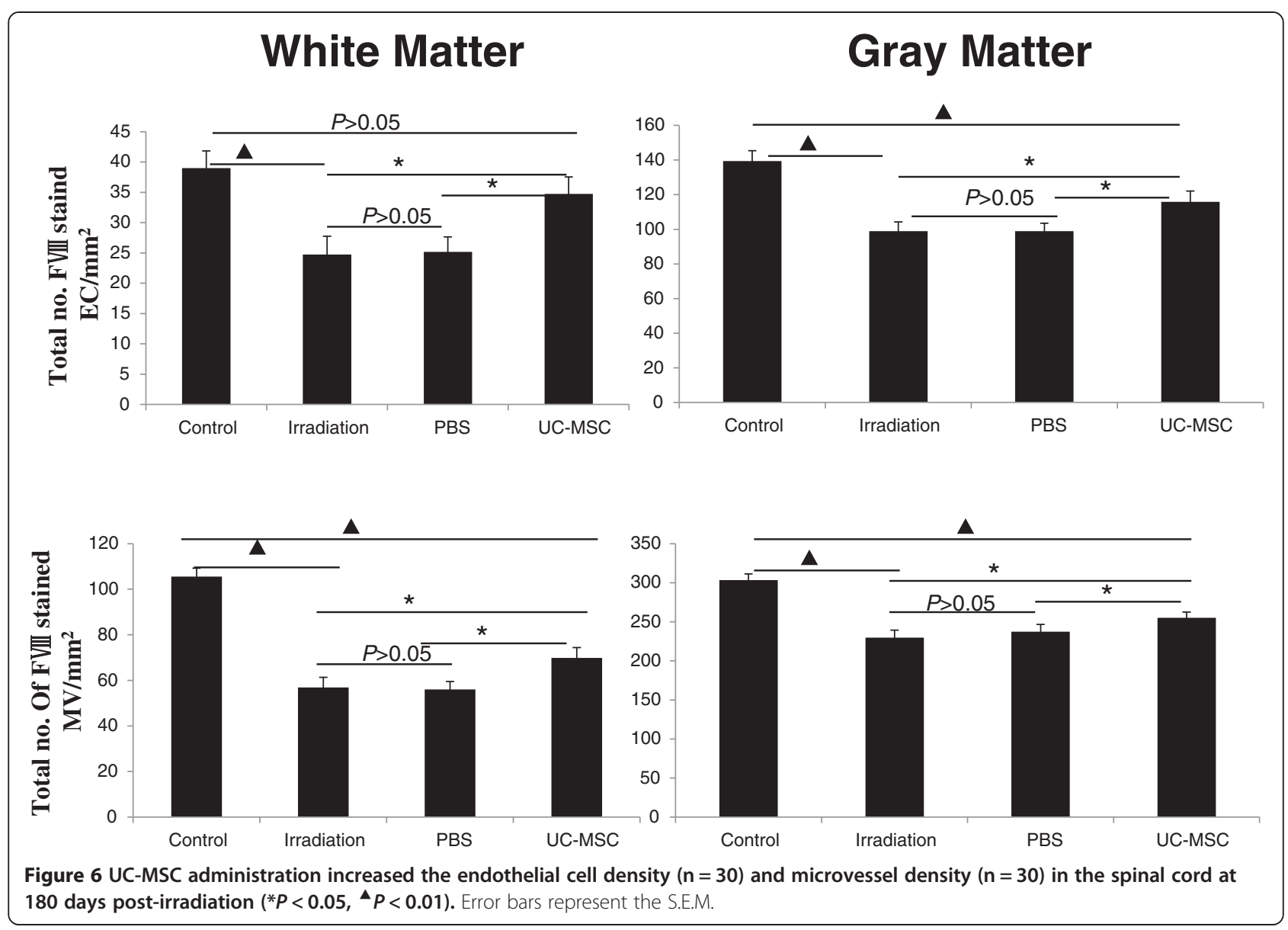

serum of the UC-MSC-treated animals were significantly lower than those in the PBS-treated animals [IL-1ß: 273.8 $\pm 13.6 \mathrm{pg} / \mathrm{ml}$; TNF- $\alpha: 117.6 \pm 5.3 \mathrm{pg} / \mathrm{ml}]$ and those in the untreated irradiated animals at 180 days post-irradiation (Figure 8). The levels of BDNF $(99.0 \pm 17.8 \mathrm{pg} / \mathrm{mg}$ ) and GDNF $(115.7 \pm 14.2 \mathrm{pg} / \mathrm{mg})$ in the spinal cord of the
UC-MSC-treated animals were significantly higher than those in the PBS-treated animals [BDNF:60.1 $\pm 6.5 \mathrm{pg} / \mathrm{mg}$; GDNF: $74.4 \pm 7.5 \mathrm{pg} / \mathrm{mg}$ ] and those in the untreated irradiated animals at 180 days post-irradiation (Figure 8). The levels of IL-1 $\beta$ and TNF- $\alpha$ in the serum and the levels of BDNF and GDNF in the spinal cord in the PBS-treated

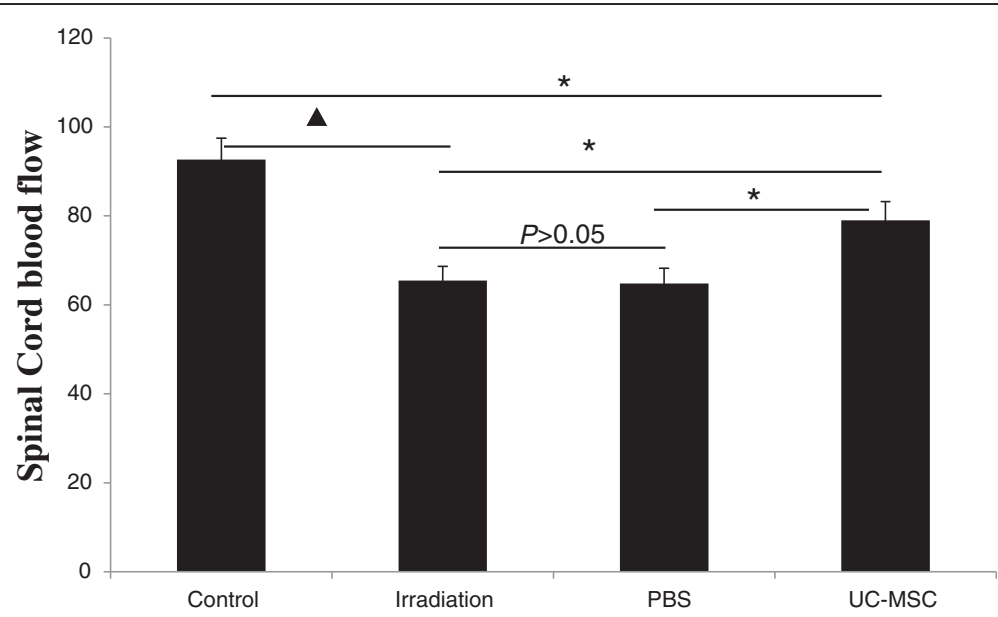

Figure 7 UC-MSC administration increased blood flow $(n=5)$ in the spinal cord at 180 days post-irradiation $\left({ }^{*} P<0.05,{ }^{\wedge} P<0.01\right)$. Error bars represent the S.E.M. 


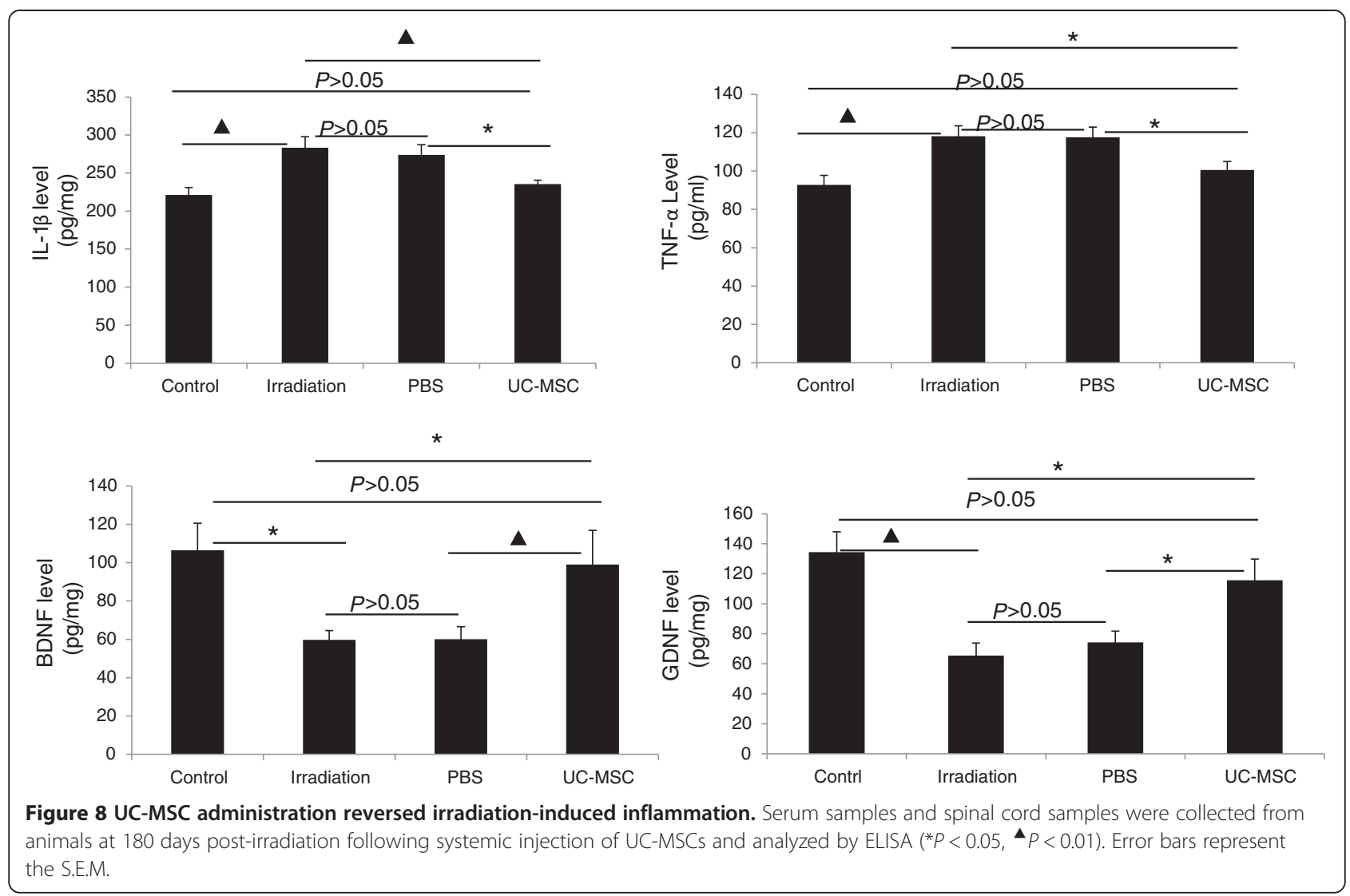

animals were similar to those in the untreated irradiated animals (Figure 8).

\section{Discussion}

The primary aim of this work was to investigate the potential beneficial effects of the delivery of UC-MSCs through the tail vein on radiation myelopathy. In the present study, using a rat model of radiation myelopathy, we demonstrated that the administration of UC-MSCs through the tail vein improved motor function, as shown by decreased forelimb paralysis; decreased histological damage in the spinal cord, as shown by Nissl staining of spinal cord sections; increased the number of neurons in the anterior horn of the spinal cord, as shown by Nissl staining; increased endothelial cell density and the microvessel density in the white matter and gray matter of the spinal cord, as determined by vWF immunohistochemistry; increased relative magnitude of spinal cord blood flow, as measured by laser-Doppler flowmetry; down-regulated pro-inflammatory cytokine expression and increased anti-inflammatory cytokine expression, as measured by an analysis of IL-1 $\beta$ and TNF- $\alpha$ levels in the serum and BDNF and GDNF levels in the spinal cord. Our data not only define the potential beneficial roles of UC-MSC infusion through the tail vein but also provide the first evidence that UC-MSCs have paracrine therapeutic effects in a rat model of radiation myelopathy.

The beneficial effects of cell-based therapies have been demonstrated using different types of cells in pre-clinical models. One of the most extensively studied cell types used for traumatic spinal cord repair in experimental models with good potential for clinical translation are MSCs [21]. However, little is known about the mechanisms underlying the potential benefits of cell grafting into the injured spinal cord [22]. The beneficial effects of MSCs have been attributed to several potential mechanisms. MSCs produce neuroprotective molecules in injured tissue [23,24], promote vascularization [24], modulate the inflammatory response $[22,25,26]$, and generate a permissive microenvironment for axonal regeneration [27-29]. In addition, MSCs have been shown to foster a positive relationship between the preservation of spinal cord parenchyma and functional and electrophysiological recovery $[23,24,27]$, and they were shown to promote axonal regeneration when transplanted after complete spinal cordtransection [29]. The therapeutic effects of immortalized neural stem cells [2] and oligodendrocyte progenitor cells (OPCs) [30] were previously determined in a rat model of radiation myelopathy. The present study is the first to examine the beneficial effects of MSCs in an animal model of radiation myelopathy. 
The therapeutic window for intervention may be a key factor that determines the beneficial effects of cell transplantation for radiation myelopathy. We applied laserDoppler flowmetry to record the relative magnitude of the spinal cord blood flow and discovered that the lowest relative magnitude of spinal cord blood flow was reached at 90 days post-irradiation [12]. Therefore, we injected UC-MSCs at 90 days post-irradiation in our rat model of radiation myelopathy, using spinal cord blood flow as a reliable indicator of the optimal time for transplantation.

The transplantation route has also been suggested to be involved in the effectiveness of cell therapy [31]. For clinical application, intravenous injection appears to be the easiest method of delivering therapeutics to the patient without risking further damage to the spinal cord [24]. Further, the efficacy and feasibility of delivering MSCs by tail vein transfusion has been confirmed $[24,32,33]$. In our initial study, we injected UC-MSCs every 7 days through the tail vein and observed that the rats died if they received an injection more than 5 times (data not shown). Thus, we injected UC-MSCs through the tail vein at 4 time points: 90, 97, 104 and 111 days post-irradiation.

Compared to MSCs from other sources, the low immunogenicity and capacity for localized immunosuppression have been well documented for UC-MSCs [16]. UC-MSCs have been shown to survive, migrate over short distances, produce large amounts of BDNF and neurotrophin-3 (NT-3) in the host spinal cord, induce fewer reactive astrocytes, increase the length of neurofilament-positive fibers and the number of growth cone-like structures around the lesion site, and enhance hindlimb locomotor function [34]. Genetic modification of UC-MSCs with NT-3 further enhanced their therapeutic effectsafter clip injury to the spinal cord [35]. Injection of Schwann-like cells induced from UC-MSCs, combined with NT-3 administration, further promoted the survival of UC-MSCs and functional recovery [36]. A combination treatment of Taxol and UC-MSCs for spinal cord injury further reduced the extent of astrocyte activation, increased axonal preservation, decreased the number of caspase-3(+) and ED-1(+) cells, and enhanced functional recovery compared with UC-MSC treatment alone [37]. Our results demonstrated that UC-MSC administration decreased irradiation-induced histological damage in the spinal cord and ultimately improved locomotion recovery. These observations confirmed that the histological improvement induced by MSCs could result in a higher degree of functional recovery.

In the present study, UC-MSCs were not detected in spinal cord sections from UC-MSC-treated animals based on immunohistochemical staining of a marker of human nuclei, MAB1281. We did not exclude the possibility that a very limited number of UC-MSCs still survived in the radiation-injured spinal cord due to some limitations of immunohistochemical technology. Reports from the early period of MSC-based cell therapy for tissue repair demonstrated that injected MSCs may survive, engraft, differentiate into specific cell types, and repair injured tissues [15]. However, subsequent studies, including the present report, supported the notion that the level of engraftment of transplanted MSCs in the host organs of recipient animals was very low. The exact mechanisms of MSC action in tissue repair are poorly understood; however, the paracrine action of MSCs has been highlighted in previous papers [16]. Direct evidence for a paracrine role of MSCs has been obtained and confirmed in studies utilizing animal models of lung [38], heart [39], and liver [40] injury, which showed that conditioned medium $(\mathrm{CM})$ generated from MSCs significantly reduced damage and stimulated regeneration in vivo. Down regulation of pro-inflammatory cytokines and up regulation of anti-inflammatory cytokines have been proposed as paracrine effects of MSCs [40]. Our results demonstrated that down regulation of the pro-inflammatory cytokines IL- $1 \beta$ and TNF- $\alpha$ in the serum occurred on a systemic level, and up regulation of the anti-inflammatory cytokines BDNF and GDNF in the spinal cord occurred on a local level. The controlled systemic and local inflammatory responses may contribute to endogenous regeneration mechanisms in the spinal cord.

MSCs have been demonstrated to increase the density of new blood vessels in traumatized spinal cord tissue, resulting in functional recovery $[24,41]$. We demonstrated that UC-MSC injection increased the endothelial cell density and the microvessel density in the white and gray matter of the spinal cord and increased spinal cord blood flow. This effect was induced by secreted factors that exert paracrine effects on local endothelial cells [42].

\section{Conclusions}

In summary, we have demonstrated that multiple injections of UC-MSCs via the tail vein significantly improved the microcirculation and microenvironment through paracrine therapeutic effects in a rat model of radiation myelopathy. To the best of our knowledge, this is the first study to evaluate MSC therapy for radiation myelopathy. The present work may provide preclinical data for the treatment of radiation myelopathy using human MSCs.

Competing interests

The authors declare that they have no competing interests.

\section{Authors' contributions}

LW (first author), JZ and XBX contributed equally to this work. LW, JZ, XBX, HXM, KZ, and WLS performed the experiments; JZ collected, analyzed and interpreted data; LW (seventh author), FL, ZLY, YL, YQW, ZFL, JNW, and WJZ contributed new reagents/analytic tools; HY conceived the study, participated in its design and coordination, obtained funding, and drafted the manuscript. All authors read and approved the final manuscript. 


\section{Acknowledgements}

This work was primarily supported by grants from the National Natural Science Foundation of China (NSFC Grant Numbers 81001220 and 81370077 to Hua You), the Affiliated Hospital of the Academy of Military Medical Sciences (to Hua You), and the 12th Five-Year Project of PLA China (to Hua You). It was partially supported by other grants from the NSFC (NSFC Grant Numbers 81202090 to Keng Zheng, 81100897 to Zhi-Fang Li, 81272914 to Feng Liang, 30972974 to Wei-Jing Zhang) and by grants from the Chongqing Science and Technology Commission (Grant Number 2013CSCT-JBKY-01703 to Li Wei). We thank Prof. Franky L. Chan at the School of Biomedical Sciences, The Chinese University of Hong Kong, Hong Kong, China for proof-reading and critical comments on the manuscript. We thank Prof. Hiroshi Kiyama at the Department of Functional Anatomy and Neuroscience, Nagoya University Graduate School of Medicine, Japan for encouragement and for providing new analytical tools. Li Wei is a recipient of a RONPAKU Fellowship (Grant ID No: CHN-11402) from the Japan Society for the Promotion of Science (JSPS) and is partially supported by an IBRO-APRC exchange fellowship.

\section{Author details}

${ }^{1}$ Affiliated Hospital of the Academy of Military Medical Sciences, No.8 East Main Street, Fengtai District, Beijing 100071, China. ${ }^{2}$ Key Laboratory of Birth Defects and Reproductive Health of the National Health and Family Planning Commission, Chongqing Population and the Family Planning Science and Technology Research Institute, Chongqing 400020, China. ${ }^{3}$ Department of Endocrine Surgery, the First Affiliated Hospital of Chongqing Medical University, Chongqing 400016, China. ${ }^{4}$ Department of Cardiology \& Institute of Clinical Medicine, Hubei University of Medicine, Shiyan, Hubei 442000, China. ${ }^{5}$ Research Institute of Surgery, Daping Hospital, Third Military Medical University, Chongqing 400042, China. ${ }^{6}$ The First Affiliated Hospital of Liaoning Medical University, No.2 Renmin Street, Guta District, Jinzhou 121000, Liaoning Province, China. PPLA 205 Hospital, No.9 Chongqing Road, Guta District, Jinzhou 121000, Liaoning Province, China.

Received: 5 July 2014 Accepted: 28 August 2014

Published: 8 September 2014

\section{References}

1. Ruifrok AC, Stephens LC, van der Koge IAJ: Radiation response of the rat cervical spinal cord after irradiation at different ages: tolerance, latency and pathology. Int J Radiat Oncol Biol Phys 1994, 29(1):73-79.

2. Rezvani M, Birds DA, Hodges H, Hopewell JW, Milledew K, Wilkinson JH: Modification of radiation myelopathy by the transplantation of neural stem cells in the rat. Radiat Res 2001, 156:408-412.

3. Daigle $\mathrm{J}$, Hong JH, Chiang CS, McBride WH: The role of tumor necrosis factor signaling pathways in the response of murine brain to irradiation. Cancer Res 2001, 61(24):8859-8865.

4. Coderre JA, Morris GM, Micca PL, Hopewell JW, Verhagen I, Kleiboer BJ, van der Kogel AJ: Late effects of radiation on the central nervous system: role of vascular endothelial damage and glial stem cell survival. Radiat Res 2006, 166(3):495-503.

5. Myers R, Rogers MA, Hornsey S: A reappraisal of the roles of glial and vascular elements in the development of white matter necrosis in irradiated rat spinal cord. Br J Cancer Suppl 1986, 7:221-223.

6. Van der Kogel AJ: Late Effects of Radiation on The Spinal Cord. In Doseeffect relationships and pathogenesis. Monograph. Rijswijk, The Netherlands: publication of the Radiobiological Institute TNO; 1979:160.

7. Van der Kogel AJ: Mechanisms of Late Injury in The Spinal Cord. In Radiation Biology in Cancer Research. Edited by Meyn RE, Withers HR. New York: Raven Press Ltd; 1980:46|-470l.

8. Van der Kogel AJ: Central Nervous System Radiation Injury in Small Animal Models. In Radiation Injury to the Nervous System. Edited by Gutin PH, Leibel SA, Sheline GE. New York: Raven Press Ltd; 1991:91-111.

9. Tofilon PJ, Fike JR: The radio response of the central nervous system: a dynamic process. Radiat Res 2000, 153(4):357-370.

10. Li YQ, Chen P, Haimovitz-Friedman A, Reilly RM, Wong CS: Endothelial apoptosis initiates acute blood-brain barrier disruption after ionizing radiation. Cancer Res 2003, 63:5950-5956.

11. Li YQ, Chen P, Jain V, Reilly RM, Wong CS: Early radiation-induced endothelial cell loss and blood-spinal cord barrier breakdown in the rat spinal cord. Radiat Res 2004, 161:143-152.
12. Zhang J, Wei L, Sun WL, Wang L, Zhang WJ, You H: Radiation-induced endothelial cell loss and reduction of the relative magnitude of the blood flow in the rat spinal cord. Brain Res 2014, 1583C:193-200.

13. Wong CS, Van der Kogel AJ: Mechanisms of radiation injury to the central nervous system: implications for neuroprotection. Mol Interv 2004, 4:273-284.

14. Salem HK, Thiemermann C: Mesenchymal stromal cells: current understanding and clinical status. Stem Cells 2010, 28(3):585-596.

15. Nasir GA, Mohsin S, Khan M, Shams S, Ali G, Khan SN, Riazuddin S: Mesenchymal stem cells and Interleukin- 6 attenuate liver fibrosis in mice. J Trans/ Med 2013, 11:78.

16. Santos JM, Bárcia RN, Simões SI, Gaspar MM, Calado S, Agua-Doce A, Almeida SC, Almeida J, Filipe M, Teixeira M, Martins JP, Graça L, Cruz ME, Cruz $\mathrm{P}$, Cruz $\mathrm{H}$ : The role of human umbilical cord tissue-derived mesenchymal stromal cells $\left(U C X^{\oplus}\right)$ in the treatment of inflammatory arthritis. J Trans/ Med 2013, 11:18.

17. Aguilar S, Nye E, Chan J, Loebinger M, Spencer-Dene B, Fisk N, Stamp G, Bonnet $D$, Janes SM: Murine but not human mesenchymal stem cells generate osteosarcoma-like lesions in the lung. Stem Cells 2007, 25(6):1586-1594

18. Yang CC, Shih YH, Ko MH, Hsu SY, Cheng H, Fu YS: Transplantation of human umbilical mesenchymal stem cells from Wharton's jelly after complete transection of the rat spinal cord. PLoS One 2008, 3(10):e3336.

19. Nordal RA, Wong CS: Intercellular adhesion molecule-1 and blood-spinal cord barrier disruption in central nervous system radiation injury. J Neuropathol Exp Neurol 2004, 63:474-483.

20. Chiang CS, Mason KA, Withers HR, McBride WH: Alteration in myelin-associated proteins following spinal cord irradiation in guinea pigs. Int J Radiat Oncol Biol Phys 1992, 24:929-937.

21. Torres-Espín A, Redondo-Castro E, Hernández J, Navarro X: Bone marrow mesenchymal stromal cells and olfactory ensheathing cells transplantation after spinal cord injury - a morphological and functional comparison in rats. Eur J Neurosci 2014, 39(10):1704-1717.

22. Torres-Espín A, Hernández J, Navarro X: Gene expression changes in the injured spinal cord following transplantation of mesenchymal stem cells or olfactory ensheathing cells. PLOS One 2013, 8(10): :76141.

23. Himes BT, Neuhuber B, Coleman C, Kushner R, Swanger SA, Kopen GC, Wagner J, Shumsky JS, Fischer I: Recovery of function following grafting of human bone marrow-derived stromal cells into the injured spinal cord. Neurorehabil Neural Repair 2006, 20(2):278-296.

24. Quertainmont R, Cantinieaux D, Botman O, Sid S, Schoenen J, Franzen R: Mesenchymal stem cell graft improves recovery after spinal cord injury in adult rats through neurotrophic and pro-angiogenic actions. PLoS One 2012, 7(6):e39500.

25. Abrams MB, Dominguez C, Pernold K, Reger R, Wiesenfeld-Hallin Z, Olson L, Prockop D: Multipotentmesenchymal stromal cells attenuate chronic inflammation and injury-induced sensitivity to mechanical stimuli in experimental spinal cord injury. Restor Neurol Neurosci 2009, 27(4):307-321.

26. Nakajima H, Uchida K, Guerrero AR, Watanabe S, Sugita D, Takeura N, Yoshida A, Long G, Wright KT, Johnson WE, Baba H: Transplantation of mesenchymal stem cells promotes an alternative pathway of macrophage activation and functional recovery after spinal cord injury. J Neurotrauma 2012, 29(8):1614-1625.

27. Hofstetter CP, Schwarz EJ, Hess D, Widenfalk J, El Manira A, Prockop DJ, Olson L: Marrow stromal cells form guiding strands in the injured spinal cord and promote recovery. Proc Natl Acad Sci U S A 2002, 99(4):2199-2204.

28. Ankeny DP, McTigue DM, Jakeman LB: Bone marrow transplants provide tissue protection and directional guidance for axons after contusive spinal cord injury in rats. Exp Neurol 2004, 190(1):17-31.

29. Koda M, Kamada T, Hashimoto M, Murakami M, Shirasawa H, Sakao S, Ino H, Yoshinaga K, Koshizuka S, Moriya H, Yamazaki M: Adenovirus vector-mediated ex vivo gene transfer of brain-derived neurotrophic factor to bone marrow stromal cells promotes axonal regeneration after transplantation in completely transected adult rat spinal cord. Eur Spine J 2007, 16(12):2206-2214.

30. Chari DM, Gilson JM, Franklin RJ, Blakemore WF: Oligodendrocyte progenitor cell (OPC) transplantation is unlikely to offer a means of preventing X-irradiation induced damage in the CNS. Exp Neurol 2006, 198(1):145-153. 
31. Dai G, Liu X, Zhang Z, Wang X, Li M, Cheng H, Hua R, Shi J, Wang R, Qin C, Gao J, An Y: Comparative analysis of curative effect of CT-guided stem cell transplantation and open surgical transplantation for sequelae of spinal cord injury. J Transl Med 2013, 11:315.

32. Kim SJ, Park KC, Lee JU, Kim KJ, Kim DG: Therapeutic potential of adipose tissue-derived stem cells for liver failure according to the transplantation routes. J Korean Surg Soc 2011, 81(3):176-186.

33. Forostyak S, Jendelova P, Sykova E: The role of mesenchymal stromal cells in spinal cord injury, regenerative medicine and possible clinical applications. Biochimie 2013, 95(12):2257-2270.

34. Hu SL, Luo HS, Li JT, Xia YZ, Li L, Zhang LJ, Meng H, Cui GY, Chen Z, Wu N, $\mathrm{Lin} J \mathrm{~K}$, Zhu G, Feng $\mathrm{H}$ : Functional recovery in acute traumatic spinal cord injury after transplantation of human umbilical cord mesenchymal stem cells. Crit Care Med 2010, 38(11):2181-2189.

35. Shang $A J$, Hong $S Q, X u Q$, Wang $H Y$, Yang $Y$, Wang $Z F, X u B N$, Jiang $X D$, Xu RX: NT-3-secreting human umbilical cord mesenchymal stromal cell transplantation for the treatment of acute spinal cord injury in rats. Brain Res 2011, 1391:102-113.

36. Guo YW, Ke YQ, Li M, Cai YQ, Jiang XD, Zhang SZ, Zhang WM, Duan CZ: Human umbilical cord-derived Schwann-like cell transplantation combined with neurotrophin-3 administration in dyskinesia of rats with spinal cord injury. Neurochem Res 2011, 36(5):783-792.

37. Zhilai Z, Hui Z, Anmin J, Shaoxiong M, Bo Y, Yinhai C: A combination of taxol infusion and human umbilical cord mesenchymal stem cells transplantation for the treatment of rat spinal cord injury. Brain Res 2012, 1481:79-89.

38. Cargnoni A, Ressel L, Rossi D, Poli A, Arienti D, Lombardi G, Parolini O: Conditioned medium from amniotic mesenchymal tissue cells reduces progression of bleomycin-induced lung fibrosis. Cytotherapy 2012, 14(2):153-161.

39. Timmers L, Lim SK, Arslan F, Armstrong JS, Hoefer IE, Doevendans PA, Piek JJ, El Oakley RM, Choo A, Lee CN, Pasterkamp G, de Kleijn DP: Reduction of myocardial infarct size by human mesenchymal stem cell conditioned medium. Stem Cell Res 2007, 1(2):129-137.

40. van Poll D, Parekkadan B, Cho CH, Berthiaume F, Nahmias Y, Tilles AW, Yarmush ML: Mesenchymal stem cell-derived molecules directly modulate hepatocellular death and regeneration in vitro and in vivo. Hepatology 2008, 47(5):1634-1643.

41. Ritfeld GJ, Nandoe Tewarie RD, Vajn K, Rahiem ST, Hurtado A, Wendell DF, Roos RA, Oudega M: Bone marrow stromal cell-mediated tissue sparing enhances functional repair after spinal cord contusion in adult rats. Cell Transplant 2012, 21(7):1561-1575.

42. Oudega M: Molecular and cellular mechanisms underlying the role of blood vessels in spinal cord injury and repair. Cell Tissue Res 2012, 349(1):269-288.

doi:10.1186/s12967-014-0246-6

Cite this article as: Wei et al: Multiple injections of human umbilical cord-derived mesenchymal stromal cells through the tail vein improve microcirculation and the microenvironment in a rat model of radiation myelopathy. Journal of Translational Medicine 2014 12:246.

\section{Submit your next manuscript to BioMed Central and take full advantage of:}

- Convenient online submission

- Thorough peer review

- No space constraints or color figure charges

- Immediate publication on acceptance

- Inclusion in PubMed, CAS, Scopus and Google Scholar

- Research which is freely available for redistribution 